\title{
Simple bedside score to optimize the time and the decision to initiate appropriate therapy for carbapenem-resistant Enterobacteriaceae
}

\author{
Violet Leibman', Emily T. Martin², Ruthy Tal-Jasper ${ }^{1}$, Leonti Grin ${ }^{3}$, Kayoko Hayakawa ${ }^{4}$, Coral Shefler ${ }^{1}$, Tal Azouri ${ }^{3}$, \\ Tamir Kaplansky ${ }^{3}$, Moran Maskit ${ }^{3}$, Tsilia Lazarovitch ${ }^{3}$, Ronit Zaidenstein ${ }^{3}$, Keith S. Kaye ${ }^{4}$ and Dror Marchaim ${ }^{1,3^{*}}$
}

\begin{abstract}
Background: Epidemiological characteristics of patients with bloodstream infections (BSI) due to extended-spectrum $\beta$-lactamase producing (ESBL) and carbapenem-resistant (CRE) strains are often similar. Mortality rates for CRE $\mathrm{BSI}$ are $70 \%$, and mean time to initiation of appropriate therapy is $\sim 5$ days. A bedside score was developed to differentiate CRE-BSIs from ESBL-BSIs, in order to help decrease the time to initiation of appropriate therapy for CRE and mortality rates.

Findings: Score was developed based of data (2007-2010) abstracted from charts of adult patients from Assaf Harofeh Medical Center (AHMC, Zeriffin, Israel), and validated on a cohort of patients from Detroit Medical Center (DMC, MI, USA). A multivariate model for presence of CRE was generated. A clinical prediction score and ROC curve was derived. 451 patients with ESBL BSIs (285 from AHMC and 166 from DMC) and 74 patients with CRE BSIs (58 from AHMC and 16 from DMC) were included. The prediction score included chemotherapy in the past 3 months (19 points), presence of foreign invasive devices (10 points), no peripheral vascular disease (10 points), reduced consciousness or cognition at time of acute illness (9 points), time in hospital prior to BSI $\geq 3$ days (7 points), and age younger than 65 years ( 6 points). A score of $\geq 32$ to define "high CRE risk" had sensitivity of $59 \%$, specificity of $76 \%$, PPV of $34 \%$ and NPV of $90 \%$.

Conclusions: The score's 90 \% NPV implies it could reduce un-necessary (and toxic) empiric use of anti-CRE therapeutics, but this should be studied prospectively and on broader populations in order to test its potential role in reducing mortality.
\end{abstract}

Keywords: CRE, KPC, ESBL, Prediction score, Nosocomial infection, Multidrug resistant

Antimicrobial resistance to broad-spectrum agents among the commonest enteric pathogens (e.g. Escherichia coli, Klebsiella pneumoniae) had become endemic in many regions worldwide [1]. Both extended-spectrum $\beta$-lactamase producing Enterobacteriaceae (ESBL) and carbapenemresistant Enterobacteriaceae (CRE) are now frequently encountered nosocomial pathogens, but there are multiple resemblances in their epidemiological features $[1,2]$. While confronted with severely septic patients, in these endemic facilities, it is often challenging for physicians to tailor the

\footnotetext{
* Correspondence: drormarchaim@gmail.com

'Sackler School of Medicine, Tel-Aviv University, Tel-Aviv, Israel

${ }^{3}$ Unit of Infectious Diseases, Assaf Harofeh Medical Center, Zerifin 70300, Israel

Full list of author information is available at the end of the article
}

most suitable empiric regimen to patients, when Enterobacteriaceae bloodstream infection (BSI) is suspected [3]. Carbapenems are still considered the agents of choice for ESBL BSIs, but are ineffective (when given alone) against CRE [3]. Since time to initiation of appropriate therapy is the strongest modifiable independent predictor for mortality in severe sepsis [4], physicians need to act fast in order to impact patients' outcomes. However, the mean number of hours to initiation of appropriate therapy for patients with CRE is $120 \pm 23$ h, i.e. 5 days [5], mainly due to delays in current routine practices for CRE determination in microbiology laboratories [1]. Therefore, it is not surprising that the attributable mortality rate among patients with CRE BSIs is $70 \%$ [6]. Physicians are 
reluctant to empirically prescribe anti-CRE agents (for severe invasive infection, i.e. BSI, polymixins are frequently and practically the only remaining appropriate agents) due to: 1) concerns pertaining to induction of resistance to the few remaining therapeutic options that are still available [7], 2) High rate of toxicities associated with polymixins use, and 3) scant controlled scientific data, pertaining to polymixins efficacy and pharmacokinetics properties in patients with CRE BSI [8].

An easy to calculate score, with high performance, based solely on parameters readily available bedside to attending clinicians, is needed in order to direct prescribers in their management of severely septic patients in the hospital settings. Prior attempts to develop such a score by our group, had few limitations, since it was based on only 16 CRE BSI cases during initial CRE emergence into the region, when endemicity was not yet established [9]. Our current study aims were to 1) develop a different bedside score, based on more cases and from a 'stable' endemic CRE region, in order to help physicians quantify the likelihood for BSI caused by CRE as opposed to ESBL, and 2) validate the score on a different cohort of patients from a distinct geographic region, that was initially studied in our first attempt for score's development [9].

\section{Findings}

The Assaf Harofeh Medical Center (AHMC) is an 813bed academic tertiary facility in the southern-central part of Israel. CRE is endemic in this region [1]. Score validation was executed at the Detroit Medical Center (DMC) health system in Southeast Michigan, which consists of 2200 inpatient beds. The Institutional Review Boards of AHMC, DMC and Wayne State University approved the study prior to its initiation. The study cohort consisted of hospitalized patients with unique episodes of bacteremia who met the following inclusion criteria: bloodstream infections (BSI) caused by either CRE or ESBL-producing Enterobacteriaceae (monomicrobial isolations), from calendar years 2007-2010, who, on the date of culture, had severe sepsis, septic shock, or multi-organ failure. Variables collected for each patient included: 1) demographics; 2) co-morbidities; 3 ) recent (3 months) exposures to antibiotics; and 4) recent ( 3 months) exposures to various healthcare-associated environments and procedures. Bacteria were identified and susceptibilities were determined in accordance with Clinical and Laboratory Standards Institute criteria [10]. Carbapenemase production screening was conducted for Enterobacteriacea which were resistant to one or more third generation cephalosporins and had elevated ertapenem MIC of $\geq 2 \mu \mathrm{g} / \mathrm{dL}$, with the modified Hodge test [10]. ESBL production was determined automatically and validated with disc diffusion tests [10]. A clinical prediction score was developed through construction of a multiple regression model for predictors of CRE BSI compared to ESBL BSI. The score was derived from the final model by multiplying the regression coefficients by a factor of 10. Score performance was assessed by calculation of sensitivity, specificity, positive predictive value (PPV), negative predictive value (NPV), and associated $95 \%$ C.I. A receiver operating characteristic (ROC) curve was generated and area under the curve (AUC) was tested against a null AUC of 0.5.

Overall, 343 patients met inclusion criteria, including 285 patients with BSIs due ESBL-producing Enterobacteriaceae and 58 patients with BSIs due to CRE. The final multivariable model of predictors for CRE BSI as compared to ESBL BSI among hospitalized adult patients at AHMC is displayed in Table 1. Based on this model, a prediction score was developed (Table 1). The score had an area under ROC curve (AUC) of 0.75 (CI-95 \% 0.690.82) (Fig. 1a). A score of $\geq 32$ to define "high CRE risk" had sensitivity of $59 \%$ (95 C.I. 45, $71 \%$ ), specificity of 76 \% (95 C.I. 71, $81 \%$ ), PPV of 34 \% (95 C.I. 25, $44 \%$ ) and NPV of $90 \%$ (95 C.I. 86, $94 \%$ ). Using a cut-point of 32 points or higher to define CRE high risk was then validated on a cohort of patients from DMC: 166 with ESBL BSIs and 16 patients with CRE BSIs. The score had an area under ROC curve of 0.64 (Fig. 1b), sensitivity of $56 \%$ ( 95 C.I. $30,80 \%$ ), specificity of $65 \%$ (95 C.I. 57, $72 \%$ ), PPV of $13 \%$ (95 C.I. 6, $24 \%$ ) and NPV of $94 \%$ (95 C.I. 88, $98 \%$ ). In a clinical setting, the score would thus be calculated by adding the number of points for each relevant patient condition (Table 1). Patients with a score less than 32 would be considered low risk for CRE, as opposed to ESBL.

This study is our first phase in trying to develop a score that can shorten the time to institution of appropriate therapy in patients with CRE BSIs, in the hope that eventually this would lead to reductions in CRE mortality rates which are reported to be $\sim 70 \%$ [6]. We know that appropriate therapy for CRE infections is delayed by $\sim 5$ days [5], and that delay of instituting appropriate therapy in severe sepsis is the strongest independent predictor for mortality [4]. Therefore, apart from improving rapid diagnostic techniques, among other optional paths, a prediction score with high performance could theoretically have a valuable role in such clinical scenarios. However, clinicians are reluctant to use prediction scores in their routine clinical practice. The score has to be simple, based only on parameters that could easily be extracted while attending the patient and reviewing the chart bedside. This is our second step in our eventual goal of developing a scientific reliable CRE BSI score for adult hospitalized patients [9]. The score was re-developed using retrospective clinical data from an endemic CRE region, constituting relatively high numbers. However, some 
Table 1 Univariable analyses and multivariable model of carbapenem-resistant Enterobacrteriaceae (CRE) bloodstream infections (BSI) compared to extended-spectrum $\beta$-lactamase producing Enterobacteriaceae (ESBL) BSI: adjusted associations and final score values

\begin{tabular}{|c|c|c|c|c|c|c|c|}
\hline \multirow[b]{2}{*}{ Variable } & \multicolumn{5}{|c|}{ Derivation Cohort (AHMC) } & \multicolumn{2}{|c|}{ Validation Cohort (DMC) } \\
\hline & $\begin{array}{l}\text { ESBL, N (\%) } \\
(N=285)\end{array}$ & $\begin{array}{l}\text { CRE, N (\%) } \\
(N=58)\end{array}$ & $\begin{array}{l}\text { OR }(95 \% \mathrm{Cl}) ; \\
p \text {-value }\end{array}$ & $\begin{array}{l}\text { AOR }{ }^{a}(95 \% \text { C.I.); } \\
p \text {-value }\end{array}$ & Score & $\begin{array}{l}\text { ESBL, N (\%) } \\
(N=166)\end{array}$ & $\begin{array}{l}\text { CRE, N (\%) } \\
(N=16)\end{array}$ \\
\hline Male gender & $169(59)$ & $29(50)$ & $0.69(0.39,1.21) ; 0.19$ & & & $91(55)$ & $7(44)$ \\
\hline Age $<65$ years & $47(17)$ & $16(28)$ & $1.9(1.00,3.71) 0.05$ & $1.8(0.81,3.92) ; 0.16$ & 6 & $78(47)$ & $5(31)$ \\
\hline LTCF residency ${ }^{\mathrm{b}}$ & $107(38)$ & $14(25)$ & $0.53(0.28,1.02) ; 0.06$ & & & $96(58)$ & $13(81)$ \\
\hline $\begin{array}{l}\text { Recent ( } 3 \text { months) hospitalization } \\
\text { or LTCF stay }\end{array}$ & $241(85)$ & $45(78)$ & $0.63(0.32,1.27) ; 0.20$ & & & $100(60)$ & $14(88)$ \\
\hline Chronic hemodialysis & $15(5)$ & $4(7)$ & $1.17(0.40,3.40) ; 0.78$ & & & $33(20)$ & $5(31)$ \\
\hline $\begin{array}{l}\text { Deteriorated functional status at } \\
\text { admission }\end{array}$ & $223(79)$ & $37(67)$ & $0.56(0.30,1.06) ; 0.07$ & & & $105(63)$ & $14(88)$ \\
\hline Congestive heart failure & $86(31)$ & $15(26)$ & $0.80(0.42,1.51) ; 0.48$ & & & $63(38)$ & $8(50)$ \\
\hline Diabetes mellitus & $115(40)$ & $22(38)$ & $0.90(0.51,1.61) ; 0.73$ & & & $86(52)$ & $13(81)$ \\
\hline Chronic renal failure ${ }^{c}$ & $97(34)$ & $15(26)$ & $0.66(0.35,1.25) ; 0.21$ & & & $68(41)$ & $10(63)$ \\
\hline No PVD ${ }^{d}$ & $218(77)$ & $50(86)$ & $1.8(0.83,4.07) ; 0.14$ & $2.7(1.06,7.02) ; 0.04$ & 10 & $134(81)$ & $13(81)$ \\
\hline $\begin{array}{l}\text { Any neurological disease (including } \\
\text { past cerebral events) }\end{array}$ & $157(55)$ & $27(47)$ & $0.7(0.4,1.3 ; 0.2$ & & & $68(41)$ & $12(75)$ \\
\hline Past or present (active) malignancy & $62(22)$ & $14(25)$ & $1.2(0.6,2.2 ; 0.7$ & & & $32(19)$ & $2(13)$ \\
\hline Hemiplegia or paraplegia & $49(17)$ & $12(21)$ & $1.27(0.63,2.58) ; 0.50$ & & & $25(15)$ & $7(44)$ \\
\hline Chemotherapy in the past 3 months & $7(2)$ & $6(10)$ & $4.53(1.46,14.0) ; 0.009$ & $6.8(1.9-24.7) ; 0.003$ & 19 & $5(3)$ & $1(6)$ \\
\hline Immunosuppression $^{e}$ & $51(18)$ & $17(29)$ & $1.88(0.99,3.57) ; 0.05$ & & & $28(17)$ & $5(31)$ \\
\hline $\begin{array}{l}\text { Any use of antibiotics in the preceding } \\
3 \text { months }\end{array}$ & $214(77)$ & $52(90)$ & $2.6(1.1,6.4) ; 0.03$ & & & $112(68)$ & $14(88)$ \\
\hline Recent (6 months) invasive procedure ${ }^{f}$ & $120(43)$ & $33(59)$ & $1.9(1.1,3.4) ; 0.03$ & & & $12(86)$ & $15(100)$ \\
\hline Intensive care unit stay at infection onset & $151(53)$ & $39(67)$ & $1.82(1.00,3.30) ; 0.05$ & & & $67(40)$ & $10(63)$ \\
\hline Permanent foreign invasive devices ${ }^{g}$ & $145(52)$ & $44(77)$ & $3.17(1.64,6.15) ; 0.001$ & $2.5(1.2-5.2) ; 0.02$ & 10 & $115(70)$ & $13(81)$ \\
\hline $\begin{array}{l}\text { Reduced consciousness and/or } \\
\text { cognition at time of acute illness }\end{array}$ & $151(53)$ & $40(73)$ & $2.33(1.23,4.41) ; 0.009$ & $2.5(1.1-5.6) ; 0.02$ & 9 & $100(60)$ & $13(81)$ \\
\hline $\begin{array}{l}\text { Severe sepsis / septic shock / } \\
\text { multiorgan failure at culture date }\end{array}$ & $133(48)$ & $36(66)$ & $2.1(1.1,3.8) ; 0.02$ & & & $43(26)$ & $4(29)$ \\
\hline $\begin{array}{l}\text { Length of hospital stay at } \mathrm{BSI}^{\mathrm{h}} \\
\text { onset }>3 \text { days }\end{array}$ & $134(47)$ & $44(76)$ & $2.85(1.49,5.43) ; 0.001$ & $1.9(0.9-4) ; 0.08$ & 7 & $61(37)$ & $11(73)$ \\
\hline
\end{tabular}

${ }^{\mathrm{a}} \mathrm{AOR}$ adjusted odds ratio. ${ }^{\mathrm{b}}$ Long-term care facility. ${ }^{\mathrm{C}}$ Serum creatinine $>1.5 \mathrm{mg} \%$ at baseline. ${ }^{\mathrm{d}}$ Peripheral Vascular Disease. ${ }^{\mathrm{e}} \mathrm{mmmunosuppression}$ include any one of the following conditions at illness onset: neutropenia $\left(<500\right.$ cells $\left./ \mathrm{mm}^{3}\right)$, glucocorticoid / steroid use in the past month, chemotherapy in the past 3 months, radiotherapy in the past 3 months, HIV, bone marrow or solid organ transplantation, or anti-TNF-a therapy in past 3 months (e.g. infliximab, adalimumab, certolizumab pegol, golimumab, etanercept). ${ }^{\mathrm{f}}$ Include any type of surgery, endoscopy, percutaneous intervention. ${ }^{9} \mathrm{An}$ invasive foreign device that was in place at least $48 \mathrm{~h}$ prior to ESBL or CRE isolation. Examples: tracheotomy, any feeding tubes, tunneled central lines, silicon-based urinary catheters, orthopedic external fixators, implanted defibrillator, pacemaker, and drains of any sort. Prosthetic heart valve or internal prosthetic joints were not considered a permanent foreign invasive devise. hbloodstream infection.

of the score components might still be unique features of the epidemiology at AHMC. The relative low performances of this suggested score, suggest larger cohorts from various endemic regions should be studied in the future. The imperfect diagnostics of CRE and ESBL production can results classification biases and should prompt consideration.

Some might argue that the clinical dilemma in today's era for attending clinicians practicing in tertiary facilities is a bit broader. For severely septic patients in the hospital setting, instituting early broad spectrum antimicrobial agents is the common standard of care [3]. For the Gram-negative bacilli (GNB) treatment arm that should be instituted, practitioners need to choose between empiric coverage of "only" multidrug resistant (MDR) GNB isolates (e.g. ESBL-producing Enterobacteriaceae, broad-spectrum cephalosporin's resistant Acinetobacter baumannii and Pseudomonas aeruginosa), as opposed to empiric coverage for extensively-drug resistant (XDR) GNBs as well (e.g. CRE, and carbapenem-resistant $A$. 


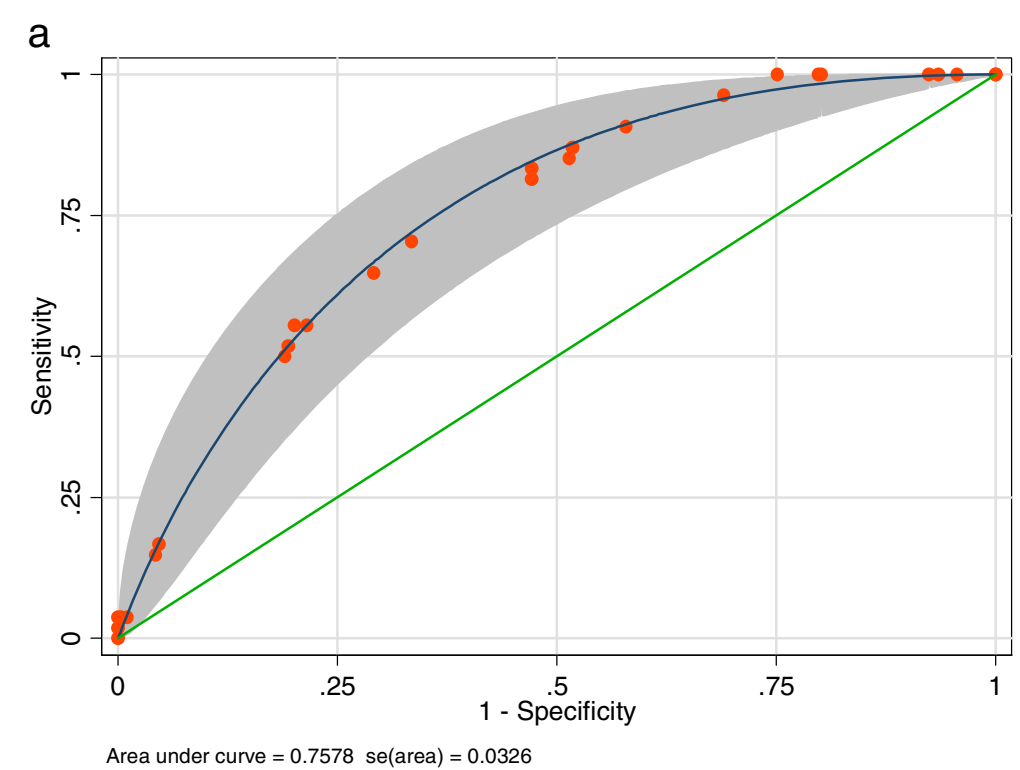

b

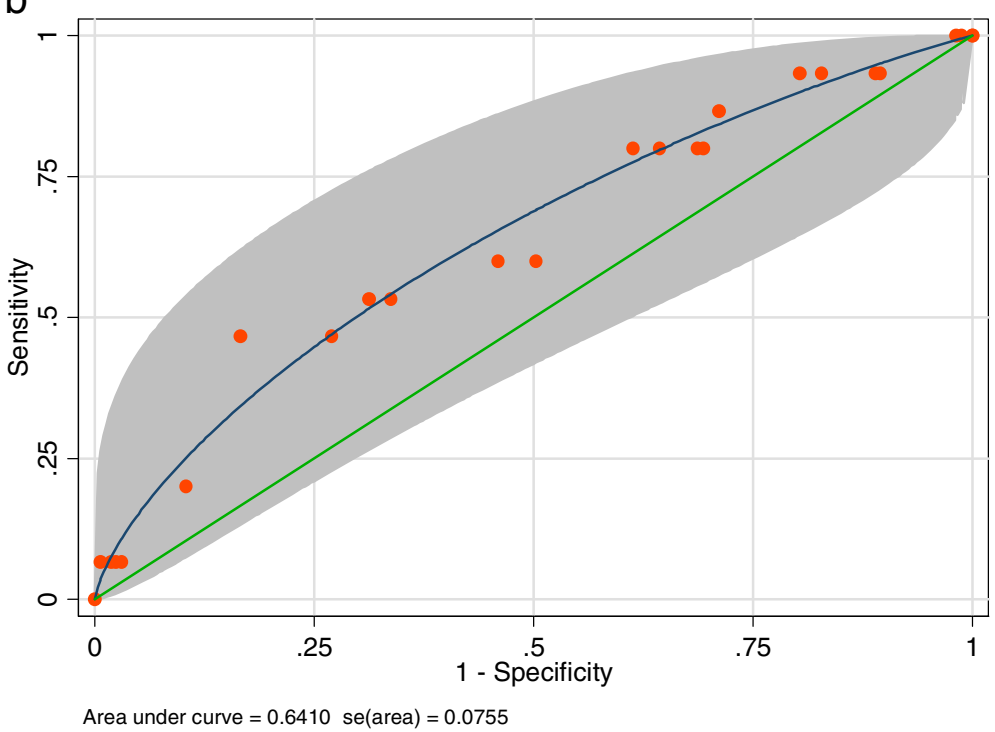

Fig. 1 a: Receiver Operating Characteristic (ROC) curve of score to predict bloodstream infection (BSI) due to carbapenem-resistant Enterobacteriaceae (CRE) as opposed to extended-spectrum $\beta$-lactamase producing Enterobacteriaceae (ESBL), Assaf Harofeh Medical Center. b: ROC curve of the score developed at Assaf Harofeh Medical Center to predict bloodstream infection due to CRE as opposed to bloodstream infection due to ESBL-producing Enterobacteriaceae at Detroit Medical Center

baumannii and P. aeruginosa) [3]. Instead of developing a "CRE score", which addresses only the Enterobacteriaceae angle, one might argue that developing a "XDR score" would be more clinically applicable. We look at this score as an initial step. Our future goals are to develop and validate both potential scores prospectively, on larger cohorts of patients, from distinct geographic locations, where both MDR and XDR GNBs are endemic in the hospital settings. This analysis is a crucial and valuable step in our effort to develop eventually a score with high performances, which could lead eventually to reductions in CRE mortality rates, while avoiding the un-necessary empiric use of broad spectrum and toxic agents (e.g. polymixins).

\section{Availability of supporting data}

The data set(s) supporting the results of this article is (are) included within the article (and its additional file(s)).

\section{Competing interests}

ETM and KSK have received research support from Pfizer, Inc. DM and KSK have received research support from Merck, Inc. 


\section{Authors' contributions}

$V L, E T M, K K$ and DM were responsible for study's design and writing the manuscript. ETM and DM were responsible for statistical analyses. RT, LG, CS, TA, TK, MM, TL, RZ participated in data collection at AHMC and KH and DM participated in data collection at DMC. All authors participated in drafting the final version of the submitted manuscript. All authors read and approved the final manuscript.

\section{Acknowledgements}

This work was performed in partial fulfillment of the M.D. thesis requirements of the Sackler Faculty of Medicine, Tel Aviv University, Tel-Aviv, Israel.

\section{Funding}

This work was supported in part by the National Institute of Health, Protocol Number 10-0065 to KSK and award K01Al099006-02 to ETM.

\section{Author details}

${ }^{1}$ Sackler School of Medicine, Tel-Aviv University, Tel-Aviv, Israel. ${ }^{2}$ Department of Epidemiology, University of Michigan School of Public Health, Ann Arbor, MI, USA. ${ }^{3}$ Unit of Infectious Diseases, Assaf Harofeh Medical Center, Zerifin 70300, Israel. ${ }^{4}$ Division of Infectious Diseases, Detroit Medical Center, Wayne State University, Detroit, MI, USA.

Received: 15 January 2015 Accepted: 25 May 2015

Published online: 04 June 2015

\section{References}

1. Schwaber MJ, Carmeli Y. Carbapenem-resistant Enterobacteriaceae: a potential threat. JAMA. 2008;300:2911-3.

2. Ben-Ami R, Rodriguez-Bano J, Arslan H, Pitout JD, Quentin C, Calbo ES, et al. A multinational survey of risk factors for infection with extended-spectrum beta-lactamase-producing enterobacteriaceae in nonhospitalized patients. Clin Infect Dis. 2009:49:682-90.

3. Peleg AY, Hooper DC. Hospital-acquired infections due to gram-negative bacteria. N Engl J Med. 2010;362:1804-13.

4. Paul M, Shani V, Muchtar E, Kariv G, Robenshtok E, Leibovici L. Systematic review and meta-analysis of the efficacy of appropriate empiric antibiotic therapy for sepsis. Antimicrob Agents Chemother. 2010;54:4851-63.

5. Ku K, Moshos J, Bheemreddy S, Wang Y, Bhargava A, Campbell M, et al. Colistin versus tigecycline for the treatment of Acinetobacter baumannii and/or carbapenem resistant Enterobacteriaceae infections. Dallas, TX, USA: Society for Healthcare Epidemiology of America (SHEA) 21st annua meeting; 2011.

6. Borer A, Saidel-Odes L, Riesenberg K, Eskira S, Peled N, Nativ R, et al. Attributable mortality rate for carbapenem-resistant Klebsiella pneumoniae bacteremia. Infect Control Hosp Epidemiol. 2009;30:972-6.

7. Bogdanovich T, Adams-Haduch JM, Tian GB, Nguyen MH, Kwak EJ, Muto CA et al. Colistin-resistant, Klebsiella pneumoniae carbapenemase (KPC)-producing Klebsiella pneumoniae belonging to the international epidemic clone ST258. Clin Infect Dis. 2011;53:373-6.

8. Garonzik SM, Li J, Thamlikitkul V, Paterson DL, Shoham S, Jacob J, et al. Population pharmacokinetics of colistin methanesulfonate and formed colistin in critically ill patients from a multicenter study provide dosing suggestions for various categories of patients. Antimicrob Agents Chemother. 2011;55:3284-94.

9. Martin ET, Tansek R, Collins V, Hayakawa K, Abreu-Lanfranco O, Chopra T, et al. The carbapenem-resistant Enterobacteriaceae score: a bedside score to rule out infection with carbapenem-resistant Enterobacteriaceae among hospitalized patients. Am J Infect Control. 2013;41:180-2.

10. CLSI. Performance standards for antimibrobial susceptibility testing. Nineteenth informational supplement. Approved standard M100-S19 Wayne. PA: Clinical and Laboratory Standards Institute; 2009.

\section{Submit your next manuscript to BioMed Central and take full advantage of:}

- Convenient online submission

- Thorough peer review

- No space constraints or color figure charges

- Immediate publication on acceptance

- Inclusion in PubMed, CAS, Scopus and Google Scholar

- Research which is freely available for redistribution

Submit your manuscript at www.biomedcentral.com/submit 